\title{
Transiting exoplanets from the CoRoT space mission
}

\section{CoRoT-6b: a transiting "hot Jupiter" planet in an 8.9d orbit around a low-metallicity star ${ }^{\star}$}

\author{
M. Fridlund ${ }^{1}$, G. Hébrard ${ }^{2}$, R. Alonso ${ }^{3,15}$, M. Deleuil ${ }^{3}$, D. Gandolfi ${ }^{10}$, M. Gillon ${ }^{15,19}$, H. Bruntt ${ }^{5}$, A. Alapini ${ }^{6}$, \\ Sz. Csizmadia ${ }^{11}$, T. Guillot ${ }^{16}$, H. Lammer ${ }^{13}$, S. Aigrain ${ }^{6,24}$, J. M. Almenara ${ }^{9,23}$, M. Auvergne ${ }^{5}$, A. Baglin ${ }^{5}$, P. Barge ${ }^{3}$, \\ P. Bordé ${ }^{4}$, F. Bouchy ${ }^{2,22}$, J. Cabrera' ${ }^{11}$, L. Carone ${ }^{12}$, S. Carpano ${ }^{1}$, H. J. Deeg ${ }^{9,23}$, R. De la Reza ${ }^{17}$, R. Dvorak ${ }^{18}$, \\ A. Erikson ${ }^{11}$, S. Ferraz-Mello ${ }^{20}$, E. Guenther ${ }^{10,9}$, P. Gondoin ${ }^{1}$, R. den Hartog ${ }^{1,7}$, A. Hatzes ${ }^{10}$, L. Jorda ${ }^{3}$, A. Léger ${ }^{4}$, \\ A. Llebaria ${ }^{3}$, P. Magain ${ }^{19}$, T. Mazeh ${ }^{8}$, C. Moutou ${ }^{3}$, M. Ollivier ${ }^{4}$, M. Pätzold ${ }^{12}$, D. Queloz ${ }^{15}$, H. Rauer ${ }^{11,21}$, D. Rouan ${ }^{3}$, \\ B. Samuel ${ }^{4}$, J. Schneider ${ }^{14}$, A. Shporer ${ }^{8}$, B. Stecklum ${ }^{10}$, B. Tingley ${ }^{9,23}$, J. Weingrill ${ }^{13}$, and G. Wuchterl ${ }^{10}$
}

(Affiliations can be found after the references)

Received 28 November 2009 / Accepted 4 January 2010

\begin{abstract}
The CoRoT satellite exoplanetary team announces its sixth transiting planet in this paper. We describe and discuss the satellite observations as well as the complementary ground-based observations - photometric and spectroscopic - carried out to assess the planetary nature of the object and determine its specific physical parameters. The discovery reported here is a "hot Jupiter" planet in an 8.9d orbit, 18 stellar radii, or 0.08 AU, away from its primary star, which is a solar-type star (F9V) with an estimated age of 3.0 Gyr. The planet mass is close to 3 times that of Jupiter. The star has a metallicity of 0.2 dex lower than the Sun, and a relatively high ${ }^{7} \mathrm{Li}$ abundance. While the light curve indicates a much higher level of activity than, e.g., the Sun, there is no sign of activity spectroscopically in e.g., the [Ca II] H\&K lines.
\end{abstract}

Key words. techniques: photometric - techniques: radial velocities - techniques: spectroscopic - planetary systems

\section{Introduction}

Transits provide insights - currently impossible to gain with other techniques - into many aspects of the physics of extrasolar planets (exoplanets) and are thus an extremely valuable tool. In spite of significant progress having been made in the detection of transiting exoplanets from the ground, e.g., Super WASP (Christian et al. 2009), the method remains significantly hampered by observations through the atmosphere and most significantly by the interruptions caused by having an orbiting rotating Earth as an observing platform. This leads to the method being most sensitive to short orbital periods (of a few days).

The spacecraft CoRoT (Convection, Rotation, and planetary Transits) was successfully launched into a near-perfect orbit on 27 December 2006. This instrument was designed to discover and study in detail exoplanets for which the transits are difficult or impossible to detect from the ground. This concerns specifically small planets or "Super-Earths" (Léger et al. 2009; Queloz et al. 2009), i.e., planets orbiting more active stars, e.g., CoRoT2b, (Alonso et al. 2008) and with longer periods, e.g., CoRoT-4b, (Aigrain et al. 2008; Moutou et al. 2008). It is relatively easy for CoRoT to detect larger planets, similar in size to Jupiter, and a number of these have also been reported (Barge et al. 2008;

* The CoRoT space mission, launched on December 27, 2006, has been developed and is being operated by CNES, with the contribution of Austria, Belgium, Brazil, ESA, The Research and Scientific Support Department of ESA, Germany and Spain.
Deleuil et al. 2008; Rauer et al. 2009). A second objective of the spacecraft is to study different aspects of micro-variability in stars, e.g., so-called p-modes in solar-type stars (Baglin et al. 2006). In this paper, we concern ourselves exclusively with the former scientific goal - transiting exoplanets - and report the discovery of the "hot Jupiter" object CoRoT-6b.

CoRoT observes from above the atmosphere, remaining pointed towards the same objects for up to longer than $150 \mathrm{~d}$ with interruptions to the light curve equalling lower than $5 \%$ (Boisnard \& Auvergne 2006). It is therefore capable of detecting transiting planets with periods in excess of 50d. With the launch of CoRoT, which provides essentially uninterrupted and long time sequences, the detection of small planets with radii only a few times the radius of the Earth has become possible (Léger et al. 2009; Queloz et al. 2009). For exoplanets, the visual magnitude range observable by CoRoT is about 11-16.5 and the photometric precision is close to the photon noise in the upper half of this range (Auvergne et al. 2009). Nevertheless, the identification of transit events is generally difficult being a demanding and complex process, which includes an ambitious follow-up program using ground-based observations (Deeg et al. 2009). CoRoT-6b is the sixth secure transiting planet detected by CoRoT. This planet is one of only a handful of transiting planets with moderately long orbital periods (currently only 5 of a total of 69 such planets have periods longer than 5 days). In this paper, we present the photometric light curve of the star, as well as the photometric and spectroscopic ground-based follow-up 
Table 1. Stellar parameters for CoRoT-6 with $U, B, V, R, \&$ I magnitudes from the Exo-dat database, and $J, H$ and $K$ magnitudes mined from the 2MASS catalogue.

\begin{tabular}{lll}
\hline \hline Parameter & Value & Error \\
\hline Corot ID & 106017681 & \\
USNO-A2 & $0900-13557622$ & \\
2MASS & $18441740+0639474$ & \\
RA (J2000) & $18^{\mathrm{h}} 44^{\mathrm{m}} 17.42$ & \\
Dec $(\mathrm{J} 2000)$ & $+6^{\circ} 39^{\prime} 47^{\prime \prime} \cdot 95$ & \\
\hline$m_{U}$ & 14.621 & 0.164 \\
$m_{B}$ & 14.638 & 0.041 \\
$m_{V}$ & 13.912 & 0.021 \\
$m_{R}$ & 13.568 & 0.02 \\
$m_{I}$ & 13.161 & 0.072 \\
$m_{J}$ & 12.518 & 0.021 \\
$m_{H}$ & 12.228 & 0.02 \\
$m_{K}$ & 12.119 & 0.023 \\
\hline
\end{tabular}

observations that establish the planetary nature of the object. We analyse the data and derive the physical properties of both the planet and its host star.

\section{Satellite observations and data processing}

\subsection{Light curve analysis}

CoRoT-6b was discovered during the third "long" observing run of CoRoT (second long observing run towards the Galactic centre direction), which took place between April 15 and September 7, 2008. This field, which is designated LRc02, has centre coordinates of approximately $\alpha=18^{\mathrm{h}} 40^{\mathrm{m}}$ and $\delta=$ $+6 \mathrm{deg}$. The planet was detected transiting the CoRoT target 0106017681 (Deleuil et al. 2009), which is an $m_{V}=13.9$ star (see Table 1). CoRoT-6b was first noticed in the so-called "alarm-mode", (Surace et al. 2008; Quentin et al. 2006), i.e., a first look at roughly processed data while a particular observing run is ongoing and relatively little data is available. The first function of this "alarm mode" is to change the time-sampling for a specific target from once every $512 \mathrm{~s}$ to once every $32 \mathrm{~s}$. The second (very important) function is to alert and initiate the follow-up campaign already during the ongoing CoRoT observations. Because of the Earth's orbital motion during a long pointing, sources observed during a run of 150 days or more, will disappear into the daytime sky very soon after the end of the CoRoT observing run. By waiting until the end of a long run, plus the time it takes to process the full data stream, the necessary follow-up observations would be delayed 5-6 months before the particular field re-emerges from behind the Sun. to be

The ephemeris of the midpoints of the eclipses was found

$T_{c}=2454595.6144 \pm 0.0002+E^{\star} \times(8.886593 \pm 0.000004),(1)$

where $E^{\star}$ is the ordered number of the transit in question.

After the detection of CoRoT-6b, the follow-up process was initiated early and the planetary nature of the object transiting CoRoT-6 was established very soon through ground-based spectroscopic observations (see below). The data presented in the current paper, however, contains the complete CoRoT light curve of the target, reduced using the latest version of the
CoRoT calibration pipeline (June 2009). This latest version (version 2.1) of the pipeline includes:

- the correction for the CCD zero offset and gain;

- a correction for the background (as measured on the sky);

- a correction for orbital effects;

- an improved spacecraft jitter correction.

The correction for the orbital effects consist of corrections for both the offset and background variations, an adjustment for pointing errors including effects on the spacecraft caused by the ingress/egress of the Earth's shadow, cross talk in the electronics, and exposure time variations. Variations in the CCD temperatures are not corrected for but these effects are very small (Auvergne et al. 2009).

According to imagery and photometry of target fields obtained with the INT/WFC before the satellite observation and stored in the Exo-dat database (Deleuil et al. 2009), we estimate that flux contamination from the background stars falling inside the CoRoT-6's photometric mask (Auvergne et al. 2009) is $2.8 \pm 0.7 \%$ of the total flux. Assuming that these contaminants are photometrically stable during the whole observing run, we subtract $2.8 \%$ of the median flux from the light curve. The estimated error in this contamination factor is taken into account during the bootstrap analysis described below.

The light curve from CoRoT for CoRoT-6 covers 144 days and comprises 331397 samples, obtained with a $\geq 95 \%$ duty cycle. In the case of the CoRoT target 0106017681, because of its relative brightness, it was selected for both fast integration (32 s) and multi-color data taking from the beginning. For a number of targets, including CoRoT-6, the photometric mask is divided into three sections ("red", "green" and "blue") and thus three light curves are obtained. To obtain a higher $S / N$, these are added together for detection purposes, whereas the colors are used mainly to exclude contaminating objects and the effect of stellar activity. In addition, the pipeline flags so-called "outliers", i.e., sudden deviations from the light curve in one integration bin. These are mostly caused by particle hits and most frequently during the satellite's passage of the South Atlantic Anomaly, where even the shielding of the focal plane is not sufficient. The outliers are simply removed from the data stream before interpretation. Long-term effects caused by particle hits leaving remnant effects on the CCD detector with timescales of seconds, minutes, and even days (see Auvergne et al. 2009) are left in the data stream by the pipeline and treated "manually" in the subsequent analysis. The different timescales of the discontinuities that are observed of course have different frequencies. Significantly long timescale excursions are much rarer events and in the present case of CoRoT-6, as can be seen by Figs. 1 and 2, are virtually non-existent. After removal of transients, the resulting light curve has a duty cycle of $\sim 92 \%$.

\subsection{Transit parameters}

We display the final light curve in Figs. 1 and 2, where we show both the complete data set covering the whole integration period as well as an enlarged portion of a section, around just one transit, which enhances the details. The final light curve shows some interesting characteristics. We first note that CoRoT-6b is an active star as are most of the other transiting planet host stars discovered by CoRoT (e.g., CoRoT-2b, $-4 \mathrm{~b} \&-7 \mathrm{~b}$ ). We detect a total of 15 transits with a maximum depth of $\sim 1.4 \%$. The transits are clearly visible in the CoRoT light curve, as can be seen from the enlarged light curve in Fig. 2. We used three different filter/model combinations to analyse the data. Initially, we 


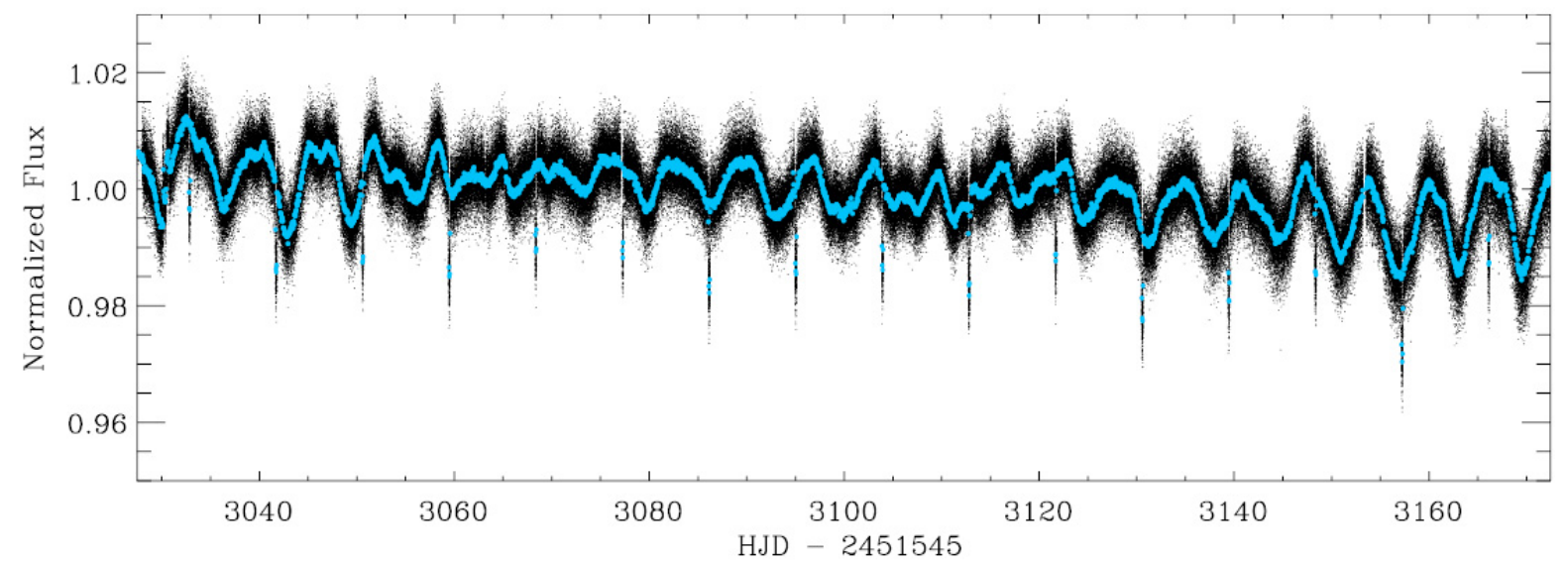

Fig. 1. The processed and normalised white light curve of the CoRoT-6 observation and the transits of the planet. It displays the total 144 days of data. The black (fuzzy) curve is the oversampled (32 s) light curve, while the overlaid (blue, sharp) curve has been re-binned to a time-sampling of $1 \mathrm{~h}$. The ordinate in the plots is HJD - 2451545, corresponding to the HJD of the first of january of 2000 (the "CoRoT date" used as a zero-point for all light curves and ephemerises). This figure clearly demonstrate the high activity of CoRoT-6.

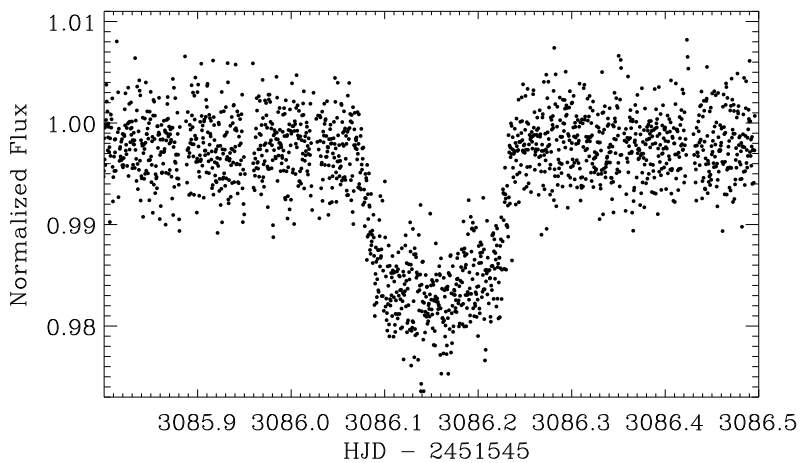

Fig. 2. Magnified portion of the processed and normalised light curve of CoRoT-6 displaying one of the transits.

used the same scheme as in some of the previous CoRoT planets (Barge et al. 2008; Alonso et al. 2008) and we refer to this scheme as the "Gimenez model", but later included alternative analyses for comparison and completion.

\subsubsection{Polynomial fit filter and Gimenez model with Amoeba-algorithm}

To extract the planetary and stellar parameters from the light curve, we use the same methodology as in Alonso et al. (2008). We recall here the main steps of the method: from the series of 15 transits, the orbital period and the transit epoch are determined by the trapezoidal fitting of the transit centres. The light curve is phase-folded to the ephemeris after performing a local linear fit to the region surrounding the transit centre to account for any local variations. The resulting light curve is displayed in Fig. 5 and has been binned in phase by $1.5 \times 10^{-4}$ in units of fraction of the orbital period. The error for each individual bin is calculated as the dispersion in the data points inside the bin, divided by the square root of the number of points per bin. The physical parameters of the star and planet are then determined through $\chi^{2}$ analysis according to the method of Giménez (2006). We model the light curve with 6 free parameters (the transit centre $\left(T_{c}\right)$, the orbital phase at first contact $\left(\theta_{1}\right)$, the radius ratio $R_{\mathrm{p}} / R_{\star}(k)$, the orbital inclination $i$, and the $u_{+}$and $u_{-}$ coefficients related to the quadratic limb-darkening coefficients $u_{a}$ and $u_{b}$ through $u_{+}=u_{a}+u_{b}$ and $u_{-}=u_{a}-u_{b}$ (Giménez 2006



Fig. 3. The sky area around CoRoT-6 (brightest star near the centre). Left: $R$-filter image with a resolution of $\sim 2^{\prime \prime}$ taken with the Euler $1.2 \mathrm{~m}$ telescope. Right: image taken by CoRoT, at the same scale and orientation. The jagged outline in its centre is the photometric aperture mask used for this object; indicated are also CoRoT's $x$ and $y$ image coordinates and positions of nearby stars from the Exo-Dat (Deleuil et al. 2009) database.

and references therein). The transit fitting is then carried out with the same method as described in detail in Barge et al. (2008) and Alonso et al. (2008), using a bootstrap analysis to fully constrain the parameter space. The results are found in Table 2. For a detailed description of the limb darkening issue, we refer the reader to the discussion reported in Deleuil et al. (2008).

As mentioned above, we detected 15 transits during the complete run . After the light curve processing, the star displayed a transit signature with a period of 8.89 days (see Table 2 ) and a total transit duration of $4.08 \pm 0.02 \mathrm{~h}$. The phase-folded transit light curve, displayed in Fig. 5, exhibits a $1 \sigma \mathrm{rms}$ noise of $4.58 \times 10^{-4}$ (458 ppm). The time sampling here is $\sim 13 \mathrm{~s}$. The results of the analysis of the final light, combined with stellar parameters derived from spectra, allow us to determine the planetary parameters. As can be seen in Sect. 3.4, stellar modeling and light curve analysis provide consistent results for the stellar mass. The light curve then implies a planetary radius of $R_{\mathrm{p}}=1.166 \pm 0.035 R_{\mathrm{Jup}}$. 
Table 2. Parameters for CoRoT- 6 and CoRoT-6b as derived from our analyses.

\begin{tabular}{llll}
\hline \hline Parameter & Value & Uncertainty & Unit \\
\hline Period & 8.886593 & 0.000004 & day \\
Epoch $\left(T_{0}\right)$ & 2454595.6144 & 0.0002 & $\mathrm{BJD}$ \\
$\theta_{1}$ & 0.00958 & $4.82057 \times 10^{-5}$ & \\
$k=R_{\mathrm{p}} / R_{\star}$ & 0.1169 & 0.0009 & \\
$i$ & 89.07 & 0.30 & degrees \\
$u_{+}$ & 0.586 & 0.068 & \\
$u_{-}$ & -0.12 & 0.13 & \\
$M_{\star}^{1 / 3} / R_{\star}$ & 0.993 & 0.018 & \\
$a / R_{\star}$ & 17.94 & 0.33 & \\
$b\left(R_{\star}\right)$ & 0.291 & 0.091 & \\
$R_{\mathrm{pl}}$ & 1.166 & 0.035 & $R_{\mathrm{Jup}}$ \\
$R_{\star}$ & 1.025 & 0.026 & $R_{\odot}$ \\
$P_{\mathrm{rot}}$ & 6.4 & 0.5 & $\mathrm{day}$ \\
$a$ & 0.0855 & 0.0015 & $\mathrm{AU}$ \\
$V_{r}$ & -18.243 & \pm 0.015 & $\mathrm{~km} \mathrm{~s}^{-1}$ \\
$e$ & $<0.1$ & & \\
$K$ & 280 & \pm 30 & $\mathrm{~m} \mathrm{~s}^{-1}$ \\
$\sigma(\mathrm{O}-\mathrm{C})$ & 57 & - & $\mathrm{m} \mathrm{s}^{-1}$ \\
$M_{\star}$ & 1.05 & \pm 0.05 & $M_{\odot}$ \\
$M_{\mathrm{p}}$ & 2.96 & \pm 0.34 & $M_{\text {Jup }}$ \\
$\rho_{\mathrm{pl}}$ & 2.32 & \pm 0.31 & $\mathrm{~g} \mathrm{~cm}^{-3}$ \\
$T_{\mathrm{eq}}$ & 1017 & \pm 19 & $\mathrm{~K}$ \\
\hline
\end{tabular}

\subsubsection{Savitzky-Golay filter and Mandel \& Agol model with harmony search algorithm}

Independent filtering and fits were also carried out. The first is as follows. Here the light curve is processed in a different, more naive way than in the "Gimenez model" (Sect. 2.2.1). After dividing the light curve by its median, a new curve was constructed by convolving the resulting light curve with a fourthorder, $4096 \times 4096$ points Savitzky-Golay filter. The standard deviation of the differences between the measured and the convolved light curves is then calculated. A $5 \sigma$ clip is applied and the whole process is repeated until we do not find any more outliers (spurious deviations from the mean $>5 \sigma$ ). A low-order (5) median-filter is then applied to increase the signal-to-noise ratio. To minimize the effect of the stellar activity, we fit the vicinity of every transit with a low order (2-3) polynomial excluding the transit points after which we divide the data with the resulting polynomial. After these steps, we fold the light curve and a binaverage is applied forming 2000 bins.

We used the Mandel \& Agol (2002) model to perform the fit to the final, phase-folded light curve. We assumed a circular orbit and a $2.8 \%$ contaminant factor. Our five parameters were: the $a / R_{\star}, k=R_{\mathrm{pl}} / R_{\star}$ ratios, the inclination and the two limb darkening coefficients $u_{a}, u_{b}$ (we apply again the quadratic limb darkening law). To find the best fit, we utilized the Harmony Search algorithm (Geem et al. 2001), which belongs to a family of genetic search algorithms. The $1 \sigma$ errors were estimated from the width of the parameter distribution to be between $\chi_{\min }^{2}$ and $\chi_{\min }^{2}+1$. The results are:

$-a / R_{\star}=17.68 \pm 0.26$

$-R_{\mathrm{pl}} / R_{\star}=0.1131 \pm 0.0013$;

$-i=89^{\circ} .19 \pm 0.25$

$-u_{+}=0.78 \pm 0.08$

$-u_{-}=0.28 \pm 0.13$.

These results are in very good agreement with the previous run ("Gimenez model"), as is clearly evident by comparing with
Table 2. The agreement is within the $3 \sigma$ error limits of both the planet-to-stellar radius ratio and the limb-darkening and within the $1 \sigma$ error bars for all the other parameters).

\subsubsection{Iterative reconstruction filter and Mandel \& Agol model with Levenberg-Marquardt algorithm}

In this model, we start with the same light curve as described in Sect. 2.1. To circumvent the issue of varying data weights associated with different time sampling, the oversampled section of the light curve was rebinned to $512 \mathrm{~s}$ sampling. Outliers were then identified and clipped out using a moving median filter (see Aigrain et al. 2009, for details). Finally, we also discarded two segments of the light curve, in the CoRoT date ranges before 3031 days, and from 3152 to 3153.54 days (see Fig. 1), corresponding to two discontinuities in the light curve. The resulting time-series was then fed into the Iterative Reconstruction Filter (IRF). A full description of this filter is given in Alapini \& Aigrain (2009), but we repeat the basic principles of the method here for completeness. The IRF treats the light curve $Y(i)$ as $Y(i)=F(i) * A(i)+R(i)$, where $A(i)$ represents the signal at the period of the planet, which is a multiplicative term applied to the intrinsic stellar flux $F(i)$, and $R(i)$ represents the observational noise. $A(i)$ is obtained by folding the light curve at the period of the transit and smoothing it using a smoothing length of 0.0006 in phase, and the original light curve is then divided with the result, which is then run through an iterative non-linear filter (Aigrain \& Irwin 2004) using a smoothing length of 0.5 days to estimate the stellar signal $F(i)$. This signal in turn is removed from the original light curve and the process is iterated until the difference in residuals from one iteration to the next remains below $10^{-8}$ for 3 consecutive iterations, which occurs after 129 iterations in this case. The IRF reconstructs all signal at the period of the transit unavoidably including stellar variability at this timescale if the star is active. This is the case for CoRoT-6, thus a 2nd order polynomial function is fitted about the transit of the phase-folded IRF-filtered light curve and divided into it. The light curve with the stellar signal removed is then fitted using the formalism of Mandel \& Agol (2002) using quadratic limb darkening to generate model transit light curves, and the IDL implementation MPFIT of the Levenberg-Marquardt fitting algorithm (Markwardt 2009) to find the best model fit to the transit light curve. The adjusted parameters are: the epoch of the centre of the first transit in the light curve T0, the planet-to-star radius ratio $R_{\mathrm{pl}} / R_{\star}$, the system scale $a / R_{\star}$, and the planet orbital inclination with respect to the plane of view. The eccentricity is fixed to zero and the limb darkening coefficients are fixed to the values in Table 2. The uncertainties in the best-fit parameters are evaluated by circularly permuting the residuals 100 times, reevaluating the parameters each time, and taking the standard deviation of the values obtained for each parameter as the uncertainty in the parameter. The planet parameters found for CoRoT- $6 \mathrm{~b}$ with the above method are

$$
\begin{aligned}
& -\mathrm{T} 0=2454595.61433 \pm 0.00017 \\
& -R_{\mathrm{pl}} / R_{\star}=0.1157 \pm 0.0006 \\
& -a / R_{\star}=17.0 \pm 0.3 \\
& -i=88.6 \pm 0.1
\end{aligned}
$$

These values are again consistent with those obtained with the other modelling schemes, although this method favours a slightly more grazing transit. 
M. Fridlund et al.: Transiting exoplanets from the CoRoT space mission. IX.

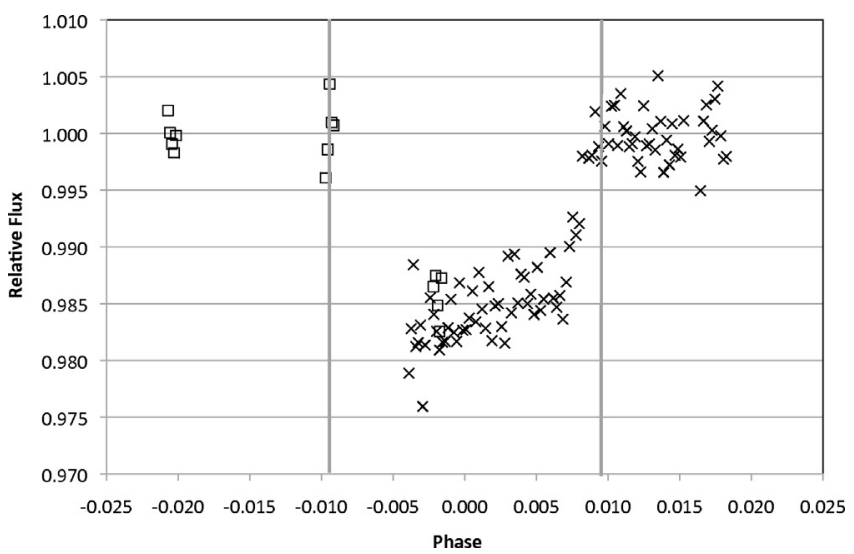

Fig. 4. Light curves of CoRoT-6 from ground-based photometric follow-up, given in normalized flux. The squares are sparse-coverage on-off photometry from the Euler telescope; the crosses are observations of an egress from WISE. They are plotted against the orbital phase; the vertical lines indicate the phase of first and last contact. The data clearly show that the transit is on the target star.

\subsubsection{Rotational modulation}

A rotational modulation with an amplitude of $\sim 2-3 \%$ is clearly present in the light curve, demonstrating clear signs of magnetic activity on the stellar photosphere. Determining the rotational period from star spot modulation is always fraught with difficulty without a proper model e.g., Bonomo et al. (2009) of the distribution of the spots on the surface and their appearance and disappearance. This is because the true evolution of star spots is poorly understood and we can imagine that, for instance, whenever a new spot appears it does so at a greater longitude than the previous one, introducing a systematic effect into the determination of the rotational period. Carrying out the required modeling is beyond the scope of the present paper and we accept that our analysis will only provide a first order approximation. We start our analysis by dividing the light curve into three roughly equal sections. From these sections, we determine rotational periods of $6.3 \pm 0.5,6.6 \pm 0.5$, and $6.4 \pm 0.4$ days respectively. We do not see any signs of systematic effects at this level of precision and simple averaging leads us then to a rotational period of $6.4 \pm 0.5$ days for this star, a value that is consistent with the value of $v \sin i$ derived from spectral analysis in Sect. 3.4 (6.9 \pm 0.9 days).

\subsection{Secondary eclipse}

Despite the relatively long period of this transiting planet, we attempted a search for its secondary eclipse. Longer period planets carry a double penalty in this aspect: first, we observe fewer eclipses for a given observation interval, causing a lower $S / N$ and secondly, the larger value of $a$ leads to a lower value of the $R_{\mathrm{pl}} / a$ ratio.

Using the same technique as Alonso et al. (2009), we calculated the $\chi^{2}$ fit using the same parameters as the observed transit, at the time of the expected secondary eclipse. The result is presented in Fig. 6. We see no trace of a possible secondary occultation. We derive a $3 \sigma$ upper limit of $1.8 \times 10^{-4}$ to the depth of the possible secondary transit in our data.

If the albedo of the planet was one, the expected depth of the secondary transit would be

$\left(\frac{R_{\mathrm{pl}}}{a}\right)^{2}=4.2 \pm 0.2 \times 10^{-5}$.

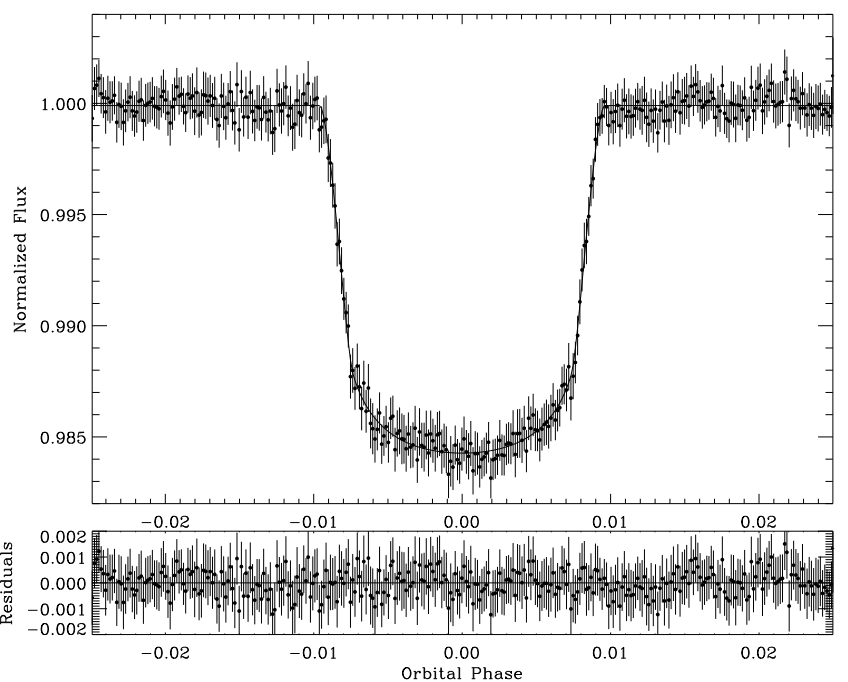

Fig. 5. The phase-folded light curve of the CoRoT-6b. The mean errorbars in this plot have been calculated as the dispersion in all the points inside the bin and divided by the square root of the number of events within each bin. The median bin size is $12.9 \mathrm{~s}$ and the resulting errorbars are $485 \mathrm{ppm}$. The dispersion inthe points outside transit is slightly higher, $500 \mathrm{ppm}$, revealing uncorrected red noise in the light curve at a low level.

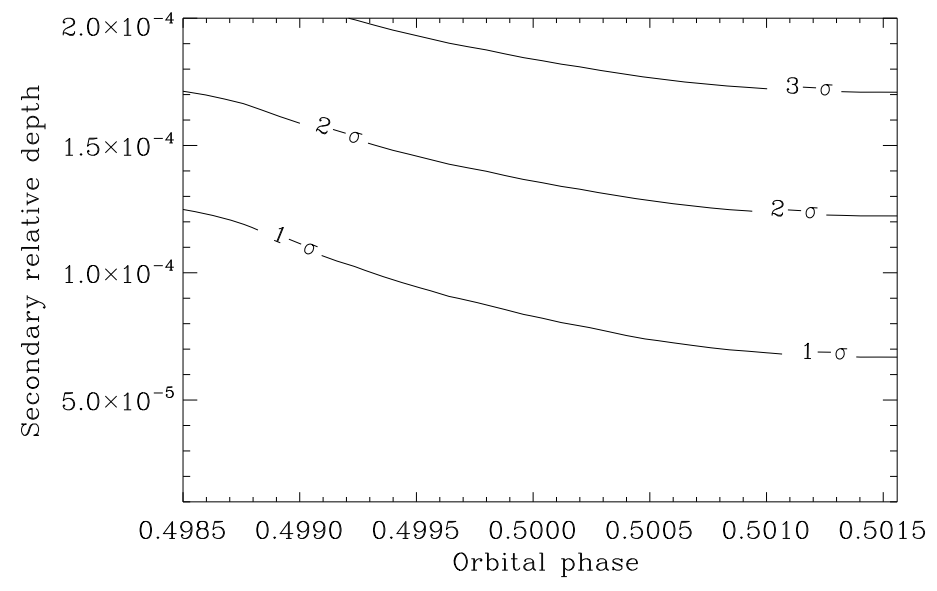

Fig. 6. The $\chi^{2}$ map of a trapezoid fit with the same parameters as the transit, in the moments of expected secondary transit. A $3 \sigma$ upper limit of $1.8 \times 10^{-4}$ to the depth of the secondary transit can be given from this plot.

Our data is thus not good enough in this case to show the secondary light.

\section{Ground based follow-up observations}

The CoRoT telescope has a large point spread function (PSF) and thus frequently background objects are found to "contaminate" the light curve obtained when integrating within a pre-set photometric mask. To exclude the possibility of spurious variation caused by such a contaminating object a comprehensive photometric (Deeg et al. 2009) and spectroscopic ground-based follow-up program is required.

The follow-up program initially consisted of observations with the radial velocity spectrograph SOPHIE (Bouchy et al. 2009; Perruchot et al. 2008), and photometric observations carried out at both the $0.46 \mathrm{~m}$ telescope at the Wise observatory in Israel and the $1.2 \mathrm{~m}$ Leonard Euler telescope at La Silla, 
Chile. These observations were initiated as soon as possible after CoRoT-6b had been identified as an exoplanetary candidate by the alarm mode.

\subsection{Photometric observations}

As mentioned above, the PSF of CoRoT is relatively large and covers typically $20^{\prime \prime} \times 10^{\prime \prime}$ on the sky. The aperture mask used to calculate the flux from the target star needs to cover all or most of the PSF (or spacecraft jitter would introduce a periodic signal and lower the precision of the actual photometry). The aperture mask will then have a significant probability of containing one or more fainter stars (see Fig. 3). The flux from these objects will thus contaminate the light curve from the target - and any variation will produce a false signal in the required data. The light curve acquired by CoRoT and believed to be the result of an transiting planet can thus instead be the result of e.g., a background eclipsing star. Using the ephemeris for transits derived from the light curve of CoRoT, observations in and outside transit were scheduled and carried out at the Euler telescope on the nights of 8-10 July, 2008, and at the Wise observatory on 23 August, 2008. In both observations, part of the transit was observed to take place on CoRoT-6, at the right time and with the right depth at mid-transit (Fig. 4). The target was thus verified as the origin of the transit. Since the activity of CoRoT-6 is similar to the amplitude of the transit signal, and the timescale is similar, likewise, this investigation proves that the activity is produced by the target.

\subsection{Radial velocity observations}

We acquired 14 spectra of the host star CoRoT- 6 between lateJune and early-September, 2008, in good weather conditions and with no strong moonlight contamination (see Table 3), at different orbital phases according to the ephemeris derived from the CoRoT photometry. These observations were performed with the SOPHIE instrument at the 1.93-m telescope of HauteProvence Observatory, France as part of the CoRoT spectroscopic follow-up (Moutou as PI). SOPHIE is a cross-dispersed, environmentally stabilized Echelle spectrograph dedicated to high-precision radial velocity measurements (Bouchy \& The Sophie Team 2006; Perruchot et al. 2008; Bouchy et al. 2009). To increase the throughput and reduce the read-out noise for this faint target, we used the high-efficiency mode (resolution power $R=40000$ ) of the spectrograph and the slow read-out mode of the $4096 \times 204815-\mu$ m-pixel CCD detector. The two optical-fiber apertures were used, the first one centred on the target and the second one on the sky. This second aperture, positioned $2^{\prime}$ away from the first one, was used to estimate the spectral pollution caused by the sky background and the moonlight, which can be quite significant in these $3^{\prime \prime}$-wide circular apertures - especially for faint targets. Exposures of a thorium-argon lamp were performed every $2-3 \mathrm{~h}$ during each observing night. They typically show drifts of around $\sim 3 \mathrm{~m} \mathrm{~s}^{-1}$, which is negligible compared to the photon noise, which is of the order of several tens $\mathrm{m} \mathrm{s}^{-1}$.

The spectra extraction and the radial velocity measurements were performed using the SOPHIE pipeline. Following the techniques described by Baranne et al. (1996) and Pepe et al. (2002), the radial velocities were measured from a weighted cross-correlation of the spectra with a numerical mask. We used a standard F0 mask that includes more than 3300 lines; cross-correlations with $\mathrm{G} 2$ and $\mathrm{K} 5$ masks gave similar results.
Table 3. Radial velocities of CoRoT-6b measured with SOPHIE.

\begin{tabular}{ccccc}
\hline \hline $\begin{array}{c}\text { BJD } \\
2454000\end{array}$ & $\begin{array}{c}R V \\
\left(\mathrm{~km} \mathrm{~s}^{-1}\right)\end{array}$ & $\begin{array}{c} \pm 1 \sigma \\
\left(\mathrm{km} \mathrm{s}^{-1}\right)\end{array}$ & $\begin{array}{c}\text { Exp. time } \\
(\mathrm{s})\end{array}$ & $\begin{array}{c}S / N \text { p. pix. } \\
(\text { at } 550 \mathrm{~nm})\end{array}$ \\
\hline 642.5591 & -18.465 & 0.066 & 2400 & 16.8 \\
646.4820 & -17.967 & 0.039 & 1805 & 20.5 \\
679.4155 & -18.345 & 0.044 & 1698 & 20.1 \\
681.4542 & -17.999 & 0.048 & 2695 & 19.0 \\
685.3983 & -18.510 & 0.050 & 1792 & 21.0 \\
689.4129 & -18.202 & 0.063 & 3600 & 26.4 \\
700.3532 & -18.011 & 0.048 & 2002 & 21.6 \\
702.3841 & -18.333 & 0.072 & 3600 & 13.4 \\
704.3410 & -18.469 & 0.044 & 2436 & 21.1 \\
706.3593 & -18.229 & 0.041 & 2400 & 22.1 \\
707.3968 & -18.041 & 0.040 & 2400 & 21.3 \\
711.3270 & -18.341 & 0.049 & 2224 & 18.1 \\
712.3234 & -18.525 & 0.060 & 2400 & 16.8 \\
715.4002 & -18.321 & 0.059 & 2974 & 15.3 \\
\hline
\end{tabular}

The resulting cross-correlation functions (CCFs) were fitted by Gaussians to determine the radial velocities and the associated photon-noise errors. The full width at half maximum of those Gaussians is $13.6 \pm 0.2 \mathrm{~km} \mathrm{~s}^{-1}$ and its contrast is $10.4 \pm 1.3 \%$ of the continuum.

Three of the 14 spectra show significant signal in the skybackground fiber, because of moonlight. This contamination was removed from CoRoT-6 spectra following the method described in Pollacco et al. (2008), Barge et al. (2008), and Hébrard et al. (2008). This induces a significant radial velocity correction for only one measurement: $-400 \pm 50 \mathrm{~m} \mathrm{~s}^{-1}$ at BJD = 2454689.4129.

The final radial velocity measurements are given in Table 3 . They are displayed in Figs. 7 and 8, along with their Keplerian fit, using the orbital period and the transit central time derived from the CoRoT photometry. The derived orbital parameters are reported in Table 2, including errorbars computed from $\chi^{2}$ variations and Monte Carlo experiments. $T_{\mathrm{eq}}$ is the zero albedo equilibrium temperature, with isotropic reemission $f=1 / 4$, and $b\left(R_{\star}\right)$ being the impact parameter. The orbit was assumed to be circular, which is a reasonable assumption for hot Jupiters. Fits with free eccentricities provide the limit $e<0.1$, without significant improvement to the dispersion in the residuals nor significant effect on the other parameters of the orbit. Two measurements were performed close to the phase $\phi=0$, but after the end of the 4-h duration transit; they should thus not be affected by the Rossiter-McLaughlin anomaly, as in for instance Bouchy et al. (2008).

The standard deviation of the residuals to the fit is $\sigma(\mathrm{O}-\mathrm{C})=57 \mathrm{~m} \mathrm{~s}^{-1}$, implying a $\chi^{2}$ of 18.8 for 12 degrees of freedom. This is slightly higher than expected, but remains in agreement with the expected errors in individual measurements, which range from \pm 39 to $\pm 72 \mathrm{~m} \mathrm{~s}^{-1}$ (see Table 3). Marginal structures are seen in the residuals of the fits on timescales similar to the orbital period of CoRoT-6b (bottom panels of Figs. 7 and 8). They could at least be partially due to stellar activity, as the CoRoT photometry exhibits a (rotational) modulation of period $6.4 \pm 0.5$ days (see Sect. 2.2). The SOPHIE coadded spectrum is of insufficient $S / N$ in the wavelength region encompassing the $\mathrm{Ca}$ II H\&K lines $(S / N \sim 6)$, so we cannot use this activity indicator. A check of the CCF bisector could reveal stellar activity or a blend with a binary as the main cause of the radial velocity variations. This check does not, however, uncover any significant dispersion in the bisector nor any trend of it changing as a function of radial velocity (see Fig. 9, upper panel). The 
M. Fridlund et al.: Transiting exoplanets from the CoRoT space mission. IX.

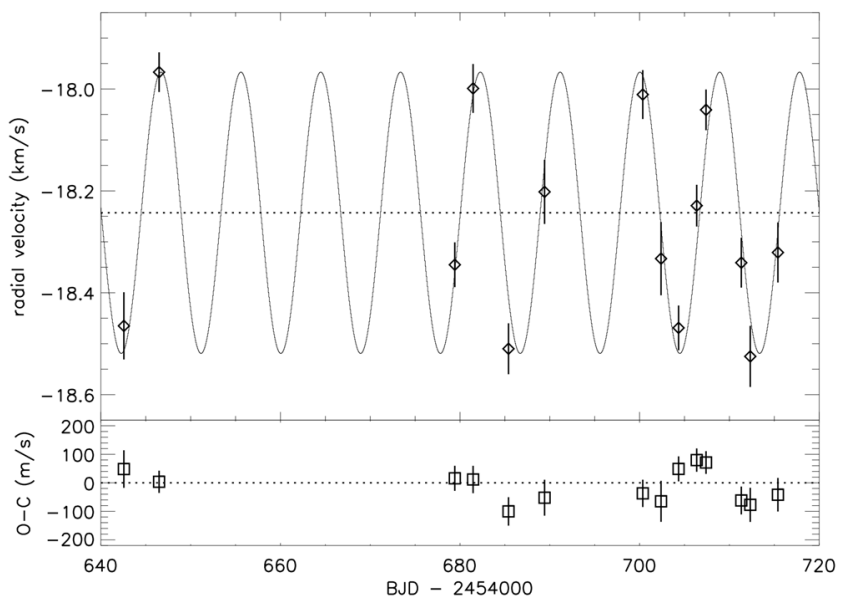

Fig. 7. Top: radial velocity SOPHIE measurements of CoRoT-6 as a function of time, and Keplerian fit to the data. The orbital parameters corresponding to this fit are reported in Table 2. Bottom: residuals of the fit with $1-\sigma$ errorbars.

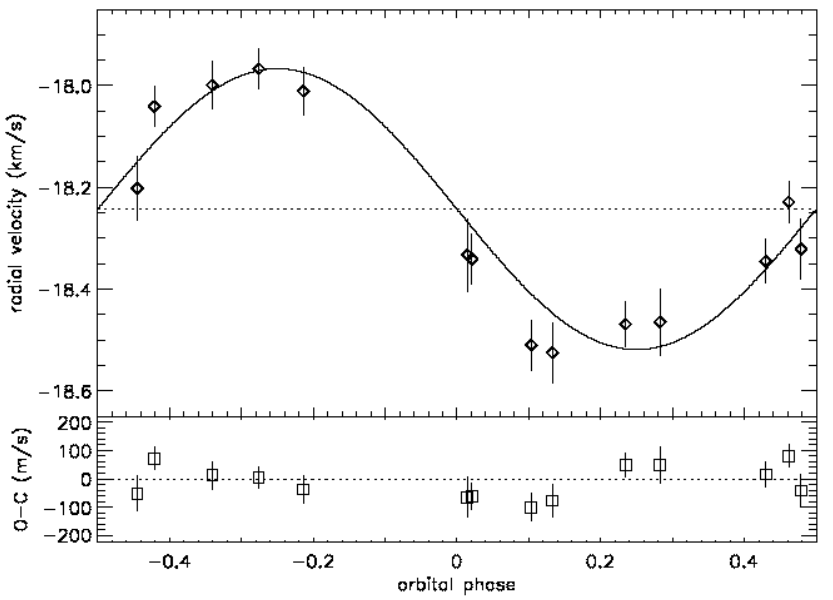

Fig. 8. Top: phase-folded radial velocity SOPHIE measurements of CoRoT-6 as a function of the orbital phase, and Keplerian fit to the data. Orbital parameters corresponding to this fit are reported in Table 2. Bottom: residuals of the fit with 1- $\sigma$ errorbars.

residuals of the fits do not either show significant anti-correlation with the bisectors (Fig. 9, lower panel), as could be expected in cases of stellar activity. This is however at the limit of our detection. These anti-correlations have allowed in some cases a subtraction of the radial velocity jitter due to stellar activity (see, e.g., Melo et al. 2007; Boisse et al. 2009), which is not possible here because of the large errorbars. The standard deviation in the bisectors is $\sigma=94 \mathrm{~m} \mathrm{~s}^{-1}$, i.e., about two times the size of the residuals. This agrees with the errorbars of the bisectors, which are two times larger than the error in the radial velocities.

Keplerian fits to the radial velocity measurements with free period and phase infer parameters that are in agreement with the CoRoT ephemeris, with, however, larger errorbars in these two parameters. The dispersion in the residuals could in that case decrease to $40 \mathrm{~m} \mathrm{~s}^{-1}$, which is slightly too low according to the errorbars in individual radial velocities.

We can thus conclude that the transit-signal detected in the CoRoT light curve could be caused by a massive planet orbiting the star. The planet CoRoT-6b induces a radial velocity
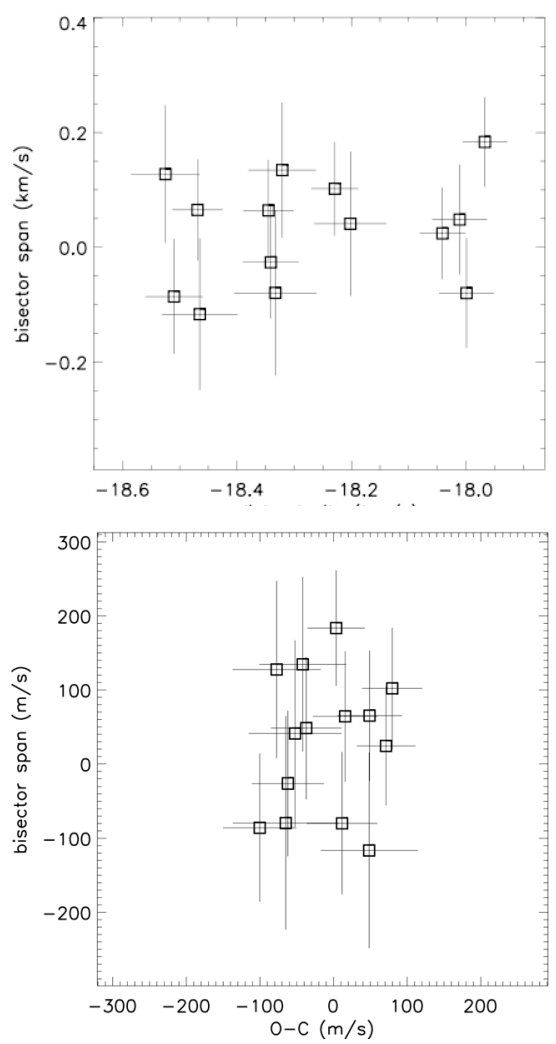

Fig. 9. Bisector span as a function of the radial velocity (top) and the radial velocity residuals after the Keplerian fit (bottom). No significant dispersion nor trend, which would have indicated activity or blend, is detected.

oscillation of its host star with a semi-amplitude $K=280 \pm$ $30 \mathrm{~m} \mathrm{~s}^{-1}$, in agreement with the photometric phase and period, $P=8.88$ days.

\subsection{Low resolution spectroscopy and spectral type classification}

To derive the spectral type of CoRoT 6 and obtain preliminary values of its physical parameters, we observed the target star with the low-resolution (LR) spectrograph mounted at the Nasmyth focus of the $2 \mathrm{~m}$ Alfred Jensch telescope of the Thüringer Landessternwarte Tautenburg (TLS). Two LR spectra covering the wavelength range 4950-7320 $\AA$ at a resolving power $R \approx 2100$ were taken on August the 3rd and 4th, 2008, under good and clear sky conditions. During each night, three consecutive exposures of 20 min were acquired to remove cosmic ray hits leading to a final $S / N \approx 80$ for each coadded spectrum. The spectral type of CoRoT 6 was derived by comparing the LR spectra of the target with a suitable grid of stellar templates, as described in Frasca et al. (2003) and Gandolfi et al. (2008). The method has proven to be quite reliable when deriving stellar temperature within one spectral subclass, pressure (or luminosity) within one class, and metallicity within $0.3-0.5$ dex. In the case of CoRoT-6, we derive a spectral type of F9, a luminosity class of $\mathrm{V}$, and a low metallicity (see method 5 in Table 6).

\subsection{High resolution spectroscopy and stellar analysis}

Because of the faintness of CoRoT-6, the SOPHIE spectra described in the last section cannot be used to carry out the stellar modeling and thus determine the fundamental physical 
Table 4. Observing log of dates and integration times of the VLT/UVES spectra.

\begin{tabular}{ll}
\hline \hline Date & Integration time $(\mathrm{s})$ \\
\hline 2008-08-27T02:43:07.087 & 2380 \\
2008-08-27T03:23:35.587 & 2380 \\
2008-09-01T00:03:51.99 & 2380 \\
2008-09-01T00:44:20.386 & 2380 \\
\hline
\end{tabular}

parameters of the host star to the required precision. One stellar parameter, the stellar density, can under ideal circumstances be obtained from a transit light curve of sufficient photometric precision (e.g., Seager \& Mallén-Ornelas 2003). Nevertheless, high-precision photometric and spectroscopic measurements carried out on other exo-planetary host stars, suggest that this rarely infers reliably to the other properties of the star - mainly because of flaws in stellar theory (Winn et al. 2008). We have therefore scheduled regular observations with the UVES spectrograph on ESO's Very Large Telescope (8.2 m) VLT to constrain the required parameters more accurately. We obtained four exposures each of $2380 \mathrm{~s}$, using a slit width of 0 !'8, which yields a resolving power of $\sim 65000$ (Table 4, ESO Program Identifier 081.C-0413(C)). The signal-to-noise ratio is $\sim 100$ over the entire range of wavelengths used in the modeling. As is now standard practice in the analysis of CoRoT stars, we use several different methods to model the stellar spectra in terms of fundamental physical parameters. Each method is applied by different teams independently.

One method uses the Spectroscopy Made Easy (SME 2.1) package (Valenti \& Piskunov 1996; Valenti \& Fischer 2005), which uses a grid of stellar models (Kurucz models or MARCS models - see below) to determine the fundamental stellar parameters iteratively. This is achieved by fitting the observed spectrum directly to the synthesized spectrum and minimizing the discrepancies using a non-linear least-squares algorithm. In addition, SME utilises input from the VALD database (Kupka et al. 1999; Piskunov et al. 1995) . The uncertainties using SME, as found by Valenti \& Fischer (2005), and based on a large sample (of more than 1000 stars) are $44 \mathrm{~K}$ in $T_{\text {eff }}, 0.06$ dex in $\log g$, and 0.03 dex in $[\mathrm{M} / \mathrm{H}]$ per measurement. However, by comparing the measurements with model isochrones they found a larger, systematic offset of $\sim 0.1$ dex and a scatter that can occasionally reach 0.3 dex, in log $g$. In CoRoT-6, we find an internal discrepancy using SME of 0.1 dex depending on which ion we use to determine $\log g$. We therefore assign $0.1 \mathrm{dex}$ as our $1 \sigma$ precision.

The second method used by us is based on the semiautomatic package VWA (Bruntt et al. 2008), which performs iterative fitting of synthetic spectra to reasonably isolated spectral lines. This method constrain $T_{\text {eff }}, \log g$, and $[\mathrm{Fe} / \mathrm{H}]$ to about $120 \mathrm{~K}, 0.13 \mathrm{dex}$, and $0.09 \mathrm{dex}$, respectively, including estimated uncertainties in the applied model atmospheres. However, larger deviations can occur for special cases when using this method (Bruntt et al. 2008).

A third method uses specific, individual models, e.g., those of Kurucz (1993) to model the $\mathrm{H} \alpha$ line and the MARCS model grid (Gustafsson et al. 2008) for the other ionic species. It uses special software to reproduce the line profiles, calculating the stellar parameters (e.g., the gravity is calculated from the $[\mathrm{Mg} \mathrm{I}]$ and [Na I] line wings). It thus does not specifically perform an iterative fitting on a grid of models. This method is described in detail in Barge et al. (2008).
Table 5. Abundance pattern in CoRoT-6 for 16 elements.

\begin{tabular}{llr|llr}
\hline \hline El. & $\log N / N_{\text {tot }}$ & $n$ & El. & $\log N / N_{\text {tot }}$ & $n$ \\
\hline Li I & +1.91 & 1 & V I & -0.19 & 2 \\
C I & -0.30 & 2 & Cr I & $-0.25 \pm 0.05$ & 7 \\
Na I & -0.30 & 2 & Cr II & $-0.22 \pm 0.07$ & 3 \\
Mg I & -0.20 & 1 & Fe I & $-0.19 \pm 0.04$ & 179 \\
Si I & $-0.22 \pm 0.04$ & 21 & Fe II & $-0.15 \pm 0.05$ & 18 \\
Si II & -0.21 & 2 & Co I & -0.01 & 2 \\
S I & -0.25 & 1 & Ni I & $-0.30 \pm 0.04$ & 37 \\
Ca I & $-0.15 \pm 0.04$ & 14 & Y II & $-0.20 \pm 0.07$ & 3 \\
Sc II & $-0.26 \pm 0.05$ & 5 & Ba II & +0.19 & 1 \\
Ti I & $-0.16 \pm 0.05$ & 13 & & & \\
Ti II & $-0.15 \pm 0.07$ & 4 & & & \\
\hline
\end{tabular}

A fourth scheme again utilises the equivalent width of a set of lines of different elements, calculating ratios. The equivalent width $(\mathrm{EW})$ of spectral lines varies as a function of temperature and can therefore be used to measure stellar temperatures $\left(T_{\text {eff }}\right)$. Using ratios of spectral lines obviates the dependency of single lines on instrumental or rotational broadening. Each temperature-calibrated line ratios can be used to derive an individual measurement of the stellar temperature $T_{\text {eff }}$. The precision in the combined temperature is improved by a factor $\sqrt{N}$, where $N$ is the number of independent measurements (Aigrain et al. 2009). The description of the calibration set of equivalentwidth line-ratios that we use here can be found in Sousa et al. (2010). The calibration consists of 433 equivalent-width lineratios (composed of 171 spectral lines of different chemical elements) calibrated in temperature with the sample of FGK stars presented in Sousa et al. (2008). This technique is automated to produce from the input stellar spectra the derivation of the stellar temperature, the equivalent width measurements being performed with the ARES software (Sousa et al. 2007) and the stellar temperature being derived using the calibrated line ratios. This technique provides a model-independent stellar spectroscopic temperature. The spectra of CoRoT-6 used here is the same as that presented earlier in Sect. 3.4. Of the 433 line-ratios, 173 line-ratios were measured and kept to derive the stellar temperature; the other line ratios were either not measured because at least one of the spectral lines was not measurable or were disregarded because the derived $T_{\text {eff }}$ was more than $2 \sigma$ away from the average $T_{\text {eff }}$ for all the line-ratios (because for example a failure of the accurate automated measurement of the equivalent width of one of the lines). When combining the 173 measurements of temperature, the stellar effective temperature obtained is $T_{\text {eff }}=6006 \pm 73 \mathrm{~K}$. This value is lower than the others in Table 6, but consistent within the errorbars.

All the methods (both low-dispersion and high-dispersion spectroscopy) utilized here allow a determination of the metallicity and we find consistent results (see Table 6). Concerning the abundances of metals, the most detailed method, however, is the VWA method (Bruntt et al. 2008), and the results can be found in Table 5 and Fig. 10. The listed uncertainties in Table 5 include the uncertainty on the atmospheric parameters $(0.04 \mathrm{dex})$ and the uncertainty on the mean value ( $>3$ lines).

The results of our analysis of CoRoT- 6 can be found in Table 6. In the case of this star, all 5 methods mentioned above have been evaluated (low- and high-resolution spectroscopy). The fact that we use several different methods to derive the fundamental stellar parameters in general, as well as the $\log g$ in particular, and reach consistent results within the applicable errors is highly satisfying. 
M. Fridlund et al.: Transiting exoplanets from the CoRoT space mission. IX.

Table 6. Stellar parameters for CoRoT- 6 derived through modeling spectra and using the 4 methods described in the text. The errors are $1 \sigma$.

\begin{tabular}{|c|c|c|c|c|c|c|c|}
\hline Method & $\begin{array}{l}T_{\text {eff }} \\
\mathrm{K}\end{array}$ & $\log g$ & {$[\mathrm{Fe} / \mathrm{H}]$} & $\begin{array}{l}V_{\text {micro }} \\
\left(\mathrm{km} \mathrm{s}^{-1}\right)\end{array}$ & $\begin{array}{l}V_{\text {macro }} \\
\left(\mathrm{km} \mathrm{s}^{-1}\right)\end{array}$ & $\begin{array}{l}v \sin i \\
\left(\mathrm{~km} \mathrm{~s}^{-1}\right)\end{array}$ & Comment \\
\hline 1. (SME 2.1) & $6090 \pm 70$ & $4.43 \pm 0.1$ & $-0.20 \pm 0.1$ & $1.2 \pm 0.10$ & $3.5 \pm 1.0$ & $7.6 \pm 1.0$ & $\begin{array}{l}\log g \text { determined from fitting } \\
\text { the line wings of } \mathrm{Mg} \mathrm{I}, \mathrm{Ca} \mathrm{I} \text { and } \mathrm{Na} \mathrm{I}\end{array}$ \\
\hline 2. (VWA) & $6090 \pm 50$ & $4.37 \pm 0.062$ & $-0.20 \pm 0.08$ & $1.24 \pm 0.10$ & $3.0 \pm 1.0$ & $8.0 \pm 1.0$ & \\
\hline 3. & $6050 \pm 100$ & $4.05 \pm 0.2$ & $-0.18 \pm 0.15$ & - & - & $7.0 \pm 1.0$ & \\
\hline 4. & $6006 \pm 73$ & - & - & - & - & - & \\
\hline 5. & $F 9^{\dagger}$ & $V^{\ddagger}$ & -0.40 to -0.10 & - & - & - & $\begin{array}{l}\text { Fitting of low } \\
\text { dispersion spectra }\end{array}$ \\
\hline
\end{tabular}

Notes. ${ }^{(\dagger)}$ Spectral type from template fitting. ${ }^{(\ddagger)}$ Luminosity class from template fitting .

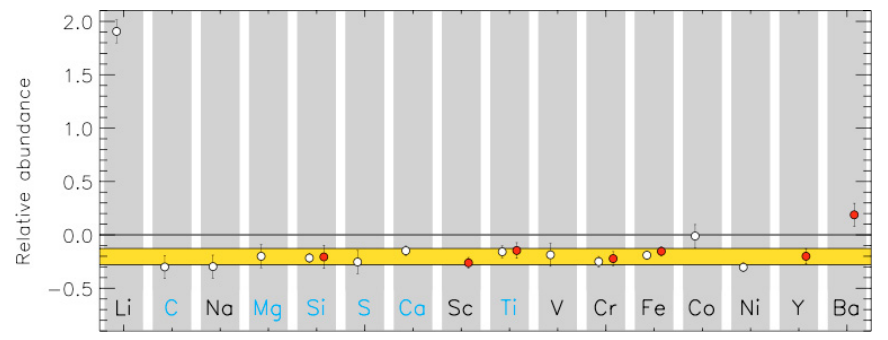

Fig. 10. Relative abundances derived from modelling the VLT/UVES and utilising the VWA package. Error bars are $1 \sigma$.

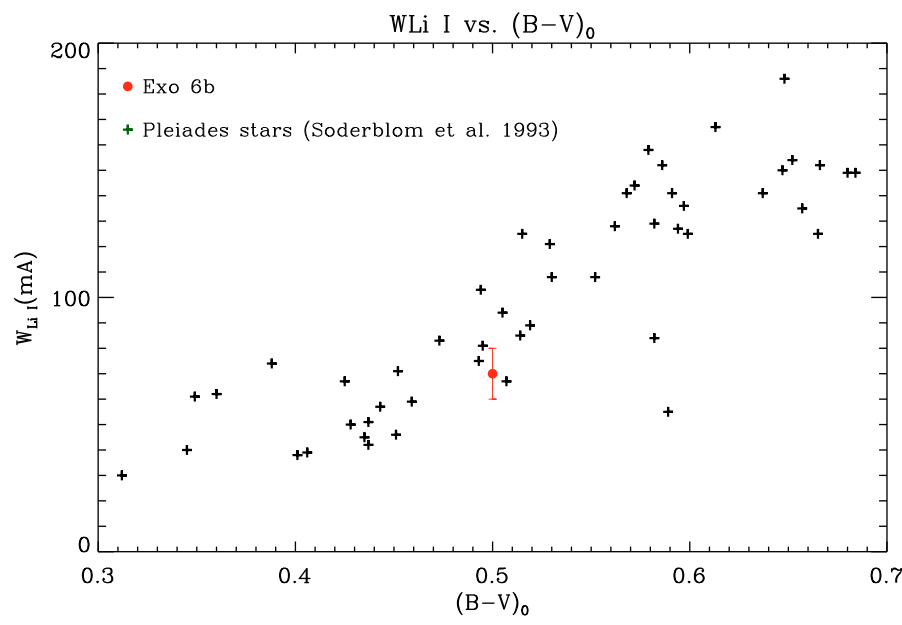

Fig. 11. The Li abundance as a function of $(B-V)_{0}$. The red marker with errorbars represents the litium abundance of CoRoT-6. The Li I equivalenth widths for Pleiades stars (Soderblom et al. 1993a) are plotted with plus symbols.

\section{Discussion and conclusion}

\subsection{Stellar parameters}

To derive the mass and radius of the parent star, we use the same methodology applied to earlier CoRoT planets (e.g., Barge et al. 2008; Bouchy et al. 2008). The light curve analysis provides $M^{1 / 3} / R_{\star}$. It is usually because this measurable provides a more robust estimate of the stellar fundamental parameters. In the case of CoRoT-6, however, we have both very good spectral information from UVES, as well as the excellent CoRoT light curve. We find that the fundamental values agree reasonably well. As discerned from the spectral analysis (Table 6), the stellar parameters are consistent with a spectral type of F9V, an effective temperature of about $6090 \pm 50 \mathrm{~K}$, a gravity compatible with a dwarf star, and a low metallicity of -0.20 (see also Table 5). From the light curve, we derive a $M^{1 / 3} / R_{\star}$ of $0.993 \pm 0.018$. Then by using the values for $M^{1 / 3} / R_{\star}$ from the light curve and $T_{\text {eff }}$ and metallicity from the spectral analysis (using STAREVOL - see Sect. 4.3), we deduce a stellar mass of $1.05 \pm 0.05 M_{\odot}$ and a stellar radius of $1.025 \pm 0.026 R_{\odot}$. Using this radius yields a $\log g$ from the light curve values of $4.44 \pm 0.023$, while the value derived from the $[\mathrm{Ca} \mathrm{I}],[\mathrm{NaI}]$, and $[\mathrm{MgI}]$ line wings in the Echelle spectrum is $4.43 \pm 0.1$ (from SME).

The metallicity of the star is quite low, $\sim-0.20$ dex. While it is generally considered that exoplanets orbit stars with metallicities greater than the one of the Sun, (Santos et al. 2003), this has been found in the very large sample of stars selected for radial velocity studies. In the sample of planets found in transit studies, however, $\sim 30 \%$ of the 53 stars for which metallicity has been published in the The Extrasolar Planets Encyclopedia catalog ${ }^{1}$ are under-abundant in metals. In a larger study, Ammler-von Eiff et al. (2009) included a consistent re-observation and analysis of 13 transiting hosts, as well as a re-analysis of about 40 other objects (including CoRoT-1b, CoRoT-2b, CoRoT-3b, and CoRoT4b), come to similar conclusions as Santos et al. (2004), i.e., that the presence of planets is a strongly rising function of metallicity. We note, however, that even in the data of Ammler-von Eiff et al. (2009), about $20 \%$ of the stars are under-abundant. As pointed out by these authors, there is probably a difference in planetary structure that depends on the metallicity of the star and reflects different formation mechanisms. We point out here that in the case of CoRoT-6b the planet is very much similar to other previously classified "hot Jupiter's".

\subsection{Planetary parameters}

There is no ambiguity in the determination of the stellar radius and thus the planetary radius is well constrained to $R_{\mathrm{p}}=$ $1.166 \pm 0.035 R_{\text {Jup }}$. Taking into account the semi-amplitude of the radial velocity curve $K=280 \pm 30 \mathrm{~m} \mathrm{~s}^{-1}$ and the orbit inclination derived from the transit $i=89.07 \pm 0.33$, the stellar mass of $M_{\star}=1.05 \pm 0.05 M_{\odot}$ translates into a planetary mass of $2.96 \pm 0.34 M_{\text {Jup }}$ for CoRoT- $6 \mathrm{~b}$. The planetary average density, $\rho_{\mathrm{pl}}$, is $2.32 \mathrm{~g} \mathrm{~cm}^{-3}$.

Comparing this latter parameter with the values for the other giant exoplanets found by CoRoT (and thus excluding CoRoT7b) and for which we have very well determined values of $\rho_{\mathrm{pl}}$, we find that the planets divide into 3 groups. In the brown dwarf mass regime, CoRoT-3b, has a very high average density of $26.4 \mathrm{~g} \mathrm{~cm}^{-3}$ demonstrating its (near) stellar status (Deleuil et al. 2008). CoRoT-1b, CoRoT-4b, and CoRoT-5b all have $0.2<\rho_{\mathrm{pl}}<0.5 \mathrm{~g} \mathrm{~cm}^{-3}$. These planets also have a mass of

${ }^{1}$ http://exoplanet.eu/catalog-transit.php 


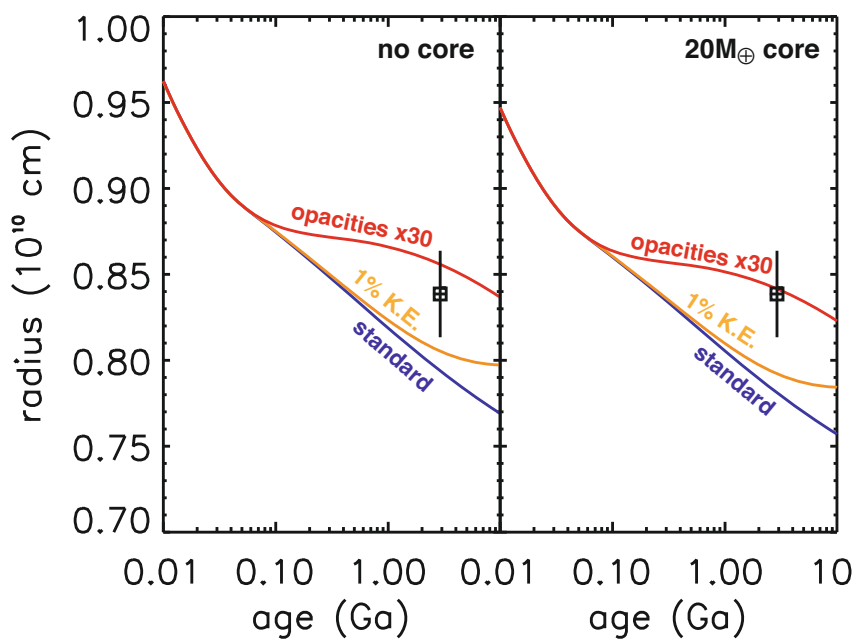

Fig. 12. Theoretical models for the contraction of CoRoT-6b with time compared to the observations. The models (Guillot 2008) assume either solar composition and no core (left panel) or the presence of a central dense core of (right panel). In each case, three possible evolution models are shown: (1) a standard model; (2) a model assuming that $1 \%$ of the incoming stellar luminosity is transformed into kinetic energy and dissipated in the deep interior by tides; (3) a model in which interior opacities have been arbitrarily increased by a factor 30 .

$0.47<M_{\mathrm{pl}}<1.03 M_{\text {Jup }}$ (Barge et al. 2008; Aigrain et al. 2008; Rauer et al. 2009). The third group consisting of CoRoT-2b and CoRoT-6b have $\rho_{\mathrm{pl}}$ of 1.3 and $2.32 \mathrm{~g} \mathrm{~cm}^{-3}$, respectively (Alonso et al. 2008). Both of these last set of planets have masses of $\sim 3 M_{\text {Jup }}$

The period of the planet orbiting CoRoT-6 is 8.9 days, which makes CoRoT-6b one of the (so far rare) transiting planets with a relatively long period ( $P \geq 5$ days). To date, only 2 of the 69 known transiting planets have periods longer then CoRoT-6b and only 6 planets have periods $\geq 5$ days, 2 of which were discovered by CoRoT. Other known transiting objects with long periods are WASP-8b with $P=8.16$ days, $M=2.23 M_{\text {Jup }}$ (Smith et al. 2009), CoRoT-4b with $P=9.20$ days, $M=0.72 M_{\text {Jup }}$ (Aigrain et al. 2008), and HD 17156 b with $P=21.22$ days, $M=$ $3.21 M_{\text {Jup }}$, (Barbieri et al. 2007; Fischer et al. 2007). There is also the case of HD $80606 \mathrm{~b}$ with $P=111.43$ days, $M=$ $3.94 M_{\text {Jup }}$ (Naef et al. 2001). Thus, of the 5 transiting objects with periods longer than 8 days, 4 have masses of between 2 and $4 M_{\text {Jup }}$, and only one - CoRoT-4b - is a somewhat smaller object of $M=0.72 M_{\text {Jup }}$. For the objects with periods between 4 and 8 days, on the other hands, 6 have masses below one $M_{\text {Jup }}, 3$ are larger, while one is the planet-brown dwarf boundary object CoRoT-3b (Deleuil et al. 2008). Of the 3 objects more massive than $1 M_{\text {Jup }}$, one barely makes the grade, another has only an upper limit to its mass and the third is more massive than $9 M_{\text {Jup }}$.

The period derived from the the CoRoT light curve is fully in phase with that derived from the radial velocity measurements. The bootstrap analysis described in Sect. 2.2 is consistent with the eccentricity of the planetary orbit being $\leq 0.1$, as derived from an analysis of the radial velocity curve (Sect. 3.2). While circularisation of planetary orbits may take quite some time (Jackson et al. 2008), we note that the age of the CoRoT-6b system derived below is consistent with a low value to the eccentricity at the distance of $0.08 \mathrm{AU}$. We used the evolutionary models of Guillot (2008) to investigate whether a planet with a relatively longer period (and thus less incident radiation) would behave differently from a more "classical" hot Jupiter. As can be seen from the results in Fig. 12, it does not. It is also a modestly inflated planet requiring: (1) a low core mass, consistent with the low-metallicity of the parent star and the star-planet metallicity correlation; and (2) either some tidal dissipation (equivalent to a few $\%$ of the incoming stellar heat flux) or an order of magnitude higher interior opacity than is generally assumed. To estimate the expected thermal evaporation of CoRoT-6b, we applied the method described in Lammer et al. (2009) and obtained mass loss rates given by $\frac{\mathrm{d} M}{\mathrm{~d} t} \sim 2 \times 10^{-8} \mathrm{~g} \mathrm{~s}^{-1}$ that are about a factor 100 lower than the loss rate of HD209458b (Yelle 2006; Penz et al. 2008). The low mass-loss rate of CoRoT-6b relative to other hot gas giants is caused by the high density of the planet and its larger orbital separation (0.08 AU). Furthermore, because of this high density, we expect the stellar EUV-induced dynamical expansion of the upper atmosphere of CoRoT-6b not to be as efficient as that of lower density close-in gas giants, hence adiabatic cooling should be less efficient. This means that a hot thermosphere-exosphere containing mainly ionized hydrogen atoms is expected. This strong ionization would enhance the planet's ability to protect itself against stellar wind erosion even in the absence of a strong planetary magnetic field (Lammer et al. 2009). Therefore, we can expect that the mass of CoRoT-6b has not changed significantly since its origin.

\subsection{Evolutionary state of the star: activity, rotation, and ${ }^{7} \mathrm{Li}$ abundance}

CoRoT-6 appears to be a solar-like star but clearly exhibits more activity, based on its light curve. It displays more rapid rotation and thus could be younger than our Sun. Unfortunately, our UVES spectrum did not cover the [Ca II] lines, although our SOPHIE data covers this wavelength region, albeit at much lower $S / N$. In spite of the very agitated state of the star discerned from photometry, we see, however, no indication of activity in the SOPHIE data. The $v \sin i$ is $7.5 \pm 1 \mathrm{~km} \mathrm{~s}^{-1}$, which indicates that the star rotates $\sim 4-5$ times more rapidly than the Sun. The 2-3\% amplitude of the rotation-modulated light curve suggests that the surface coverage by photospheric spots on CoRoT-6 is significantly higher than on the Sun. This high level of magnetic activity is most likely to be linked to its rapid rotation. High activity and rapid rotation are considered to be signs of stellar youth (Simon et al. 1985; Guedel et al. 1997; Soderblom et al. 2001) because both rotation and magnetic activity in late-type dwarfs continue to decrease as the star evolves unless the star's rotation is tidally locked in a close binary system. It has also been suggested by e.g., Skumanich (1972) that the Li surface abundance decreases with stellar evolution. Hence, another index of stellar youth is provided by the Li I absorption line of $75 \mathrm{m \AA}$ equivalent width that we detected in the spectrum of CoRoT-6, indicating a ${ }^{7} \mathrm{Li}$ abundance $\left(\log N / N_{\text {tot }}\right)$ of +1.91 (Table 5$)$.

Based on the $\mathrm{Li}$ abundance for the appropriate type of star and the results of Sestito \& Randich (2005), we estimate the age of CoRoT-6 to be between 2.5 and 4.0 Gyr. It is however worth noting that solar-type stars reach the zero-age main sequence (ZAMS) rotating at a variety of rates, from roughly solar on up to 100 times that velocity, as seen, for example, in the Pleiades (Stauffer \& Hartmann 1987; Soderblom et al. 1993b,a). A wide range of $\mathrm{Li}$ abundances are also found in the Pleiades, and data for CoRoT-6 can actually be included into the range of Li-values found for that cluster, although all other indications are that the CoRoT-6 is significantly older than the Pleiades (see Fig. 11). Li depletion has also been found in several $\mathrm{T}$ Tauri stars at levels inconsistent with their young ages (Magazzu et al. 1992). Hence, while magnetic activity, rapid rotation, and elevated Li abundance may be signs of stellar youth, the converse 
is not necessarily true. Furthermore, stars of lower metallicity have a shallower outer convection zone. This means that lithium lasts longer in these stars, mainly because the distance between the convection zone and the nuclear destruction region is larger (Castro et al. 2009). In the case of CoRoT-6, the high ${ }^{7} \mathrm{Li}$ abundance could be explained by the low metallicity, even in a relatively mature star. This should also be viewed in terms of the results of Israelian et al. (2009), who determined a relation between the presence of planets and an under-abundance of ${ }^{7} \mathrm{Li}$, the explanation being that the presence of a planet could increase the amount of mixing and deepen the convective zone to such an extent that the Li can be burned. In the case of CoRoT-6, the planet is relatively far away and it could thus be that the effect is negligible in this case.

We have also used evolutionary models of STAREVOL (Palacios, private communication) and CESAM (Morel \& Lebreton 2008), and using the $M^{1 / 3} / R_{\star}$ relation and stellar radius of $1.02 R_{\odot}$ (Table 2 - see above) to calculate the age of CoRoT-6, obtaining a value between 1 and 3.3 Gyr. Combining this value with the ${ }^{7} \mathrm{Li}$ analysis above would restrict the age to a range between 2.5 and 3.3 Gyr. In view of the rapid rotation and large level of activity, we would be inclined to favor the lower value but there is no information in our data that requires this.

\section{Summary}

The CoRoT space mission has discovered its sixth transiting planet, designated CoRoT-6b. In summary, we conclude that:

- A planet of $2.96 M_{\text {Jup }}$ orbits the star CoRoT 0106017681.

- Its orbital period is $\sim 8.9$ days and the ephemeris for the transits is compatible with a circular orbit $(e \leq 0.1)$. The planets radius is $1.166 R_{\text {Jup. }}$.

- CoRoT-6b has a density of $2.32 \mathrm{~g} \mathrm{~cm}^{-3}$, similar to that of CoRoT-2b but higher than the lower mass objects CoRoT$1 b$, CoRoT-4b, and CoRoT-5b.

- The star is solar-like with a spectral type of F9V, a mass of $1.05 M_{\odot}$, and a stellar radius of $1.025 R_{\odot}$. Its metallicity is relatively low ( -0.20 dex $)$ compared to other planet-hosting stars.

- We assign an age of 1-3.3 Gyr, based on evolutionary tracks. The higher end of this range is consistent with the high ${ }^{7} \mathrm{Li}$ abundance, given the paucity of other metals.

- No secondary eclipse is observed in the CoRoT data. This is consistent with expectations, becasue of the greater distance from the star in this case than for e.g., either CoRoT-1b and CoRoT-2b.

- The discovery of CoRoT-6b clearly demonstrates the capability of CoRoT to discover and study long-period transiting planets.

Acknowledgements. We are grateful to N. Piskunov of Uppsala Astronomical Observatory for making SME available to us, and for answering questions about its implementation and operation.

The team at IAC acknowledges support by grant ESP2007-65480-C02-02 of the Spanish Ministerio de Ciencia e Innovación.

The building of the input CoRoT/Exoplanet catalog (Exo-dat) was made possible thanks to observations collected for years at the Isaac Newton Telescope (INT), operated on the island of La Palma by the Isaac Newton group in the Spanish Observatorio del Roque de Los Muchachos of the Instituto de Astrosica de Canarias.

The German CoRoT team (TLS and University of Cologne) acknowledges DLR grants 50OW0204, 50OW0603, and 50QP07011.

We are also grateful to an anonymous referee whose very constructive comments have helped us produce a more stringent paper.

\section{References}

Aigrain, S., \& Irwin, M. 2004, MNRAS, 350, 331

Aigrain, S., Collier Cameron, A., Ollivier, M., et al. 2008, A\&A, 488, L43

Aigrain, S., Pont, F., Fressin, F., et al. 2009, A\&A, 506, 425

Alapini, A., \& Aigrain, S. 2009, MNRAS, 397, 1591

Alonso, R., Aigrain, S., Pont, F., Mazeh, T., \& The CoRoT Exoplanet Science Team 2009, in IAU Symp., 253, 91

Alonso, R., Auvergne, M., Baglin, A., et al. 2008, A\&A, 482, L21

Ammler-von Eiff, M., Santos, N. C., Sousa, S. G., et al. 2009, A\&A, 507, 523

Auvergne, M., Bodin, P., Boisnard, L., et al. 2009, A\&A, 506, 411

Baglin, A., Michel, E., Auvergne, M., \& The COROT Team 2006, in Proc. of SOHO 18/GONG 2006/HELAS I, Beyond the spherical Sun, ESA SP-624

Baranne, A., Queloz, D., Mayor, M., et al. 1996, A\&AS, 119, 373

Barbieri, M., Alonso, R., Laughlin, G., et al. 2007, A\&A, 476, L13

Barge, P., Baglin, A., Auvergne, M., et al. 2008, A\&A, 482, L17

Boisnard, L., \& Auvergne, M. 2006, in ESA SP-1306, ed. M. Fridlund,

A. Baglin, J. Lochard, \& L. Conroy, 19

Boisse, I., Moutou, C., Vidal-Madjar, A., et al. 2009, A\&A, 495, 959

Bonomo, A. S., Aigrain, S., Bordé, P., et al. 2009, A\&A, 495, 647

Bouchy, F., \& The Sophie Team. 2006, in Tenth Anniversary of 51 Peg-b:

Status of and prospects for hot Jupiter studies, ed. L. Arnold, F. Bouchy, \& C. Moutou, 319

Bouchy, F., Queloz, D., Deleuil, M., et al. 2008, A\&A, 482, L25

Bouchy, F., Hébrard, G., Udry, S., et al. 2009, A\&A, 505, 853

Bruntt, H., De Cat, P., \& Aerts, C. 2008, A\&A, 478, 487

Castro, M., Vauclair, S., Richard, O., et al. 2009, A\&A, 494, 663

Christian, D. J., Gibson, N. P., Simpson, E. K., et al. 2009, MNRAS, 392, 1585

Deeg, H. J., Gillon, M., Shporer, A., et al. 2009, A\&A, 506, 343

Deleuil, M., Deeg, H. J., Alonso, R., et al. 2008, A\&A, 491, 889

Deleuil, M., Meunier, J. C., Moutou, C., et al. 2009, AJ, 138, 649

Fischer, D. A., Vogt, S. S., Marcy, G. W., et al. 2007, ApJ, 669, 1336

Frasca, A., Alcalá, J. M., Covino, E., et al. 2003, A\&A, 405, 149

Gandolfi, D., Alcalá, J. M., Leccia, S., et al. 2008, ApJ, 687, 1303

Geem, Z. W., Kim, J. H., \& Lonatathan, G. V. 2001, Simulation, 76, 60

Giménez, A. 2006, A\&A, 450, 1231

Guedel, M., Guinan, E. F., \& Skinner, S. L. 1997, ApJ, 483, 947

Guillot, T. 2008, Phys. Sc. T, 130, 014023

Gustafsson, B., Edvardsson, B., Eriksson, K., et al. 2008, A\&A, 486, 951

Hébrard, G., Bouchy, F., Pont, F., et al. 2008, A\&A, 488, 763

Israelian, G., Delgado Mena, E., Santos, N. C., et al. 2009, Nature, 462, 189

Jackson, B., Greenberg, R., \& Barnes, R. 2008, ApJ, 678, 1396

Kupka, F., Piskunov, N., Ryabchikova, T. A., Stempels, H. C., \& Weiss, W. W. 1999, A\&AS, 138, 119

Kurucz, R. 1993, SYNTHE Spectrum Synthesis Programs and Line

Data. Kurucz CD-ROM No. 18. Cambridge, Mass.: Smithsonian Astrophysical Observatory

Lammer, H., Odert, P., Leitzinger, M., et al. 2009, A\&A, 506, 399

Léger, A., Rouan, D., Schneider, J., et al. 2009, A\&A, 506, 287

Magazzu, A., Rebolo, R., \& Pavlenko, I. V. 1992, ApJ, 392, 159

Mandel, K., \& Agol, E. 2002, ApJ, 580, L171

Markwardt, C. B. 2009, in ASP Conf. Ser. 411, ed. D. A. Bohlender, D. Durand, \& P. Dowler, 251

Melo, C., Santos, N. C., Gieren, W., et al. 2007, A\&A, 467, 721

Morel, P., \& Lebreton, Y. 2008, Ap\&SS, 316, 61

Moutou, C., Bruntt, H., Guillot, T., et al. 2008, A\&A, 488, L47

Naef, D., Latham, D. W., Mayor, M., et al. 2001, A\&A, 375, L27

Penz, T., Erkaev, N. V., Kulikov, Y. N., et al. 2008, Planet. Space Sci., 56, 1260

Pepe, F., Mayor, M., Galland, F., et al. 2002, A\&A, 388, 632

Perruchot, S., Kohler, D., Bouchy, F., et al. 2008, in SPIE Conf. Ser., 7014

Piskunov, N. E., Kupka, F., Ryabchikova, T. A., Weiss, W. W., \& Jeffery, C. S. 1995, A\&AS, 112, 525

Pollacco, D., Skillen, I., Collier Cameron, A., et al. 2008, MNRAS, 385, 1576

Queloz, D., Bouchy, F., Moutou, C., et al. 2009, A\&A, 506, 303

Quentin, C. G., Barge, P., Cautain, R., et al. 2006, in ESA SP-1306, ed. M. Fridlund, A. Baglin, J. Lochard, \& L. Conroy, 409

Rauer, H., Queloz, D., Csizmadia, S., et al. 2009, A\&A, 506, 281

Santos, N. C., Israelian, G., Mayor, M., Rebolo, R., \& Udry, S. 2003, A\&A, 398, 363

Santos, N. C., Israelian, G., \& Mayor, M. 2004, A\&A, 415, 1153

Seager, S., \& Mallén-Ornelas, G. 2003, ApJ, 585, 1038

Sestito, P., \& Randich, S. 2005, A\&A, 442, 615

Simon, T., Herbig, G., \& Boesgaard, A. M. 1985, ApJ, 293, 551

Skumanich, A. 1972, ApJ, 171, 565

Smith, A. M. S., Hebb, L., Collier Cameron, A., et al. 2009, MNRAS, 398, 1827

Soderblom, D. R., Jones, B. F., Balachandran, S., et al. 1993a, AJ, 106, 1059

Soderblom, D. R., Stauffer, J. R., Hudon, J. D., et al. 1993b, ApJS, 85, 315

Soderblom, D. R., Jones, B. F., \& Fischer, D. 2001, ApJ, 563, 334 
Sousa, S. G., Santos, N. C., Israelian, G., Mayor, M., \& Monteiro, M. J. P. F. G. 2007, A\&A, 469, 783

Sousa, S. G., Santos, N. C., Mayor, M., et al. 2008, A\&A, 487, 373

Sousa, S. G., Alapini, A., Israelian, G., \& Santos, N. C. 2010, A\&A, 512, A13

Stauffer, J. R., \& Hartmann, L. W. 1987, ApJ, 318, 337

Surace, C., Alonso, R., Barge, P., et al. 2008, in SPIE Conf., 7019

Valenti, J. A., \& Piskunov, N. 1996, A\&AS, 118, 595

Valenti, J. A., \& Fischer, D. A. 2005, ApJS, 159, 141

Winn, J. N., Holman, M. J., Torres, G., et al. 2008, ApJ, 683, 1076

Yelle, R. V. 2006, Icarus, 183, 508

1 Research and Scientific Support Department, European Space Agency, Keplerlaan1, 2200AG, Noordwijk, The Netherlands e-mail: malcolm. fridlund@esa.int

2 Institut d'Astrophysique de Paris, UMR7095 CNRS, Université Pierre \& Marie Curie, 98bis boulevard Arago, 75014 Paris, France

3 LAM, UMR 6110, CNRS/Univ. de Provence, 38 rue F. Joliot-Curie, 13388 Marseille, France

4 Institut d'Astrophysique Spatiale, Université Paris XI, 91405 Orsay, France

5 LESIA, Observatoire de Paris-Meudon, 5 place Jules Janssen, 92195 Meudon, France

6 School of Physics, University of Exeter, Exeter, EX4 4QL, UK

7 Netherlands Institute for Space Research, SRON, Sorbonnelaan 2, 3584 CA, Utrecht, The Netherlands

8 Wise Observatory, Tel Aviv University, Tel Aviv 69978, Israel
9 Instituto de Astrofísica de Canarias , 38205 La Laguna, Tenerife, Spain

10 Thüringer Landessternwarte, 07778 Tautenburg, Germany

11 Institute of Planetary Research, DLR, 12489 Berlin, Germany

12 Rheinisches Institut für Umweltforschung an der Universität zu Köln, Aachener Strasse 209, 50931 Köln, Germany

13 Space Research Institute, Austrian Academy of Science, Schmiedlstr. 6, 8042 Graz, Austria

14 LUTH, Observatoire de Paris-Meudon, 5 place Jules Janssen, 92195 Meudon, France

15 Observatoire de Genève, Université de Genève, 51 chemin des Maillettes, 1290 Sauverny, Switzerland

16 Université de Nice Sophia Antipolis, CNRS, Observatoire de la Côte d'Azur, BP 4229, 06304 Nice, France

17 Observatório Nacional, Rio de Janeiro, RJ, Brazil

18 Institute for Astronomy, University of Vienna, Türkenschanzstrasse 17, 1180 Vienna, Austria

19 IAG Université du Liège, Allée du 6 aôut 17, Liège 1, Belgium

20 IAG Universidade de Sao Paulo, Sao Paulo, Brasil

21 TU Berlin, Zentrum für Astronomie und Astrophysik, Hardenbergstr. 36, 10623 Berlin, Germany

22 Observatoire de Haute-Provence, CNRS/OAMP, 04870 St Michel l'Observatoire, France

23 Dept. de Astrofísica, Universidad de La Laguna, Tenerife, Spain

24 Oxford Astrophysics, University of Oxford, Keble Road, Oxford OX1 3RH, UK 